\title{
Whole cell biosynthesis of a functional oligosaccharide, 2'-fucosyllactose, using engineered Escherichia coli
}

\author{
Won-Heong Lee ${ }^{1,2,4}$, Panchalee Pathanibul', Josh Quarterman', Jung-Hyun Jo ${ }^{4}$, Nam Soo Han ${ }^{3}$, Michael J Miller ${ }^{1}$, \\ Yong-Su Jin ${ }^{1,2^{*}}$ and Jin-Ho Seo ${ }^{4^{*}}$
}

\begin{abstract}
Background: 2'-Fucosyllactose (2-FL) is a functional oligosaccharide present in human milk which protects against the infection of enteric pathogens. Because 2-FL can be synthesized through the enzymatic fucosylation of lactose with guanosine 5'-diphosphate (GDP)-L-fucose by a-1,2-fucosyltransferase (FuCT2), an 2-FL producing Escherichia coli can be constructed through overexpressing genes coding for endogenous GDP-L-fucose biosynthetic enzymes and heterologous fucosyltransferase.
\end{abstract}

Results: The gene for FucT2 from Helicobacter pylori was introduced to the GDP-L-fucose producing recombinant E. coli BL21 star(DE3) strain. However, only small amount of 2-FL was produced in a batch fermentation because the E. coli BL21star(DE3) strain assimilated lactose instead of converting to 2-FL. As an alternative host, the E. coli JM109(DE3) strain which is incapable of assimilating lactose was chosen as a 2-FL producer. Whole cell biosynthesis of 2-FL from lactose was investigated in a series of batch fermentations using various concentrations of lactose. The results of batch fermentations showed that lactose was slowly assimilated by the engineered E. coli JM109(DE3) strain and 2-FL was synthesized without supplementation of another auxiliary sugar for cell growth. A maximum 2-FL concentration of $1.23 \mathrm{~g} / \mathrm{l}$ was obtained from a batch fermentation with $14.5 \mathrm{~g} / \mathrm{l}$ lactose. The experimentally obtained yield (g 2-FL/g lactose) corresponded to $20 \%$ of the theoretical maximum yield estimated by the elementary flux mode (EFM) analysis.

Conclusions: The experimental 2-FL yield in this study corresponded to about 20\% of the theoretical maximum yield, which suggests further modifications via metabolic engineering of a host strain or optimization of fermentation processes might be carried out for improving 2-FL yield. Improvement of microbial production of 2-FL from lactose by engineered $E$. coli would increase the feasibility of utilizing 2-FL as a prebiotic in various foods.

Keywords: Recombinant Escherichia coli, GDP-L-fucose, a-1,2-fucosyltransferase, 2'-fucosyllactose, Elementary flux mode analysis

\section{Background}

Human milk oligosaccharides (HMOs) are known to be the most relevant factor for the development of intestinal microbiota in breast-fed infants [1]. Also, HMOs have been reported to play important roles in preventing adhesion of pathogens and toxins to epithelial surfaces [2]. Fucosyloligosaccharides, such as $2^{\prime}$-fucosyllactose, lacto-

\footnotetext{
* Correspondence: ysjin@illinois.edu; jhseo94@snu.ac.kr

${ }^{1}$ Department of Food Science and Human Nutrition, University of Illinois at Urbana-Champaign, Urbana IL 61801, USA

${ }^{2}$ Institute for Genomic Biology, University of Illinois at Urbana-Champaign, Urbana IL 61801, USA

Full list of author information is available at the end of the article
}

$N$-fucopentaose and lacto- $N$-difucohexaose, are common HMOs. Fucosylated oligosaccharides act as growth stimulating factors for select Bifidobacteria and soluble analogs of receptors for pathogenic bacteria, thereby protecting infants against infection from enteric pathogens and binding of toxins $[3,4]$. Specifically, $\alpha-1,2$-linked fucosylated oligosaccharides are reported to exhibit protective activity against several pathogens including Campylobacter jejuni [3,5], Salmonella enteric serotype Typhimurium [6], Enterotoxigenic E. coli [7], Helicobacter pylori [8] and noroviruses [9]. Among them, 2'-fucosyllactose (2-FL) is the most abundant fucosyloligosaccharide in human milk and accounts for more than $30 \%$ of total HMOs [3,5]. Low 
levels of 2-FL in the milk of sore mothers have been reported to be associated with a higher rate of diarrhea in breast-fed infants [3]. Hence, 2-FL is a promising oligosaccharide for nutraceutical and pharmaceutical purposes.

2-FL can be synthesized through the enzymatic fucosylation of lactose by $\alpha-1,2$ fucosyltransferase (FucT2), which requires guanosine $5^{\prime}$-diphosphate (GDP)-L-fucose as a donor of L-fucose [10]. Escherichia coli is known to be able to synthesize GDP-L-fucose since GDP-L-fucose is used for biosynthesis of colanic acid, one of the main components of the cell wall [11]. Therefore, 2-FL can be produced via engineering of the GDP-L-fucose biosynthetic pathway and overexpression of the fucosyltransferase gene in metabolically engineered E. coli. Figure 1 shows the metabolic pathway for biosysnthesis of GDP-L-fucose and 2-FL in recombinant $E$. coli.

Previously, biosynthesis of fucosyloligosaccharides using a recombinant microorganism and fucosyltransferase has been reported. The enzymatic synthesis of 2-FL was examined by using purified FucT2, GDP-L-fucose and lactose [10], however, the high cost of GDP-L-fucose and FucT2 purification may be a limiting factor for large-scale production of fucosyloligosaccharides. Production of several fucose-containing lacto-oligosaccharides in recombinant $E$. coli was also reported through simultaneous overexpression of fucosyltransferase and the regulatory protein for colanic acid biosynthesis [12,13], which suggested that whole cell synthesis of fucosyloligosaccharides through direct amplification of the GDP-L-fucose biosynthesis might be feasible.
To construct an efficient 2-FL production system by metabolic engineering, an understanding and detailed analysis of a cellular metabolic network involved in the 2-FL biosynthesis is important. Elementary flux mode (EFM) analysis has emerged as a powerful tool for metabolic pathway analysis. EFM analysis is a useful mathematical tool for defining and describing all metabolic routes that are both stoichiometrically and thermodynamically feasible for a group of enzymes. The EFM analysis can decompose a complex metabolic network of many highly interconnected reactions into uniquely organized pathways that support steady state of metabolism. EFM analysis can provide identification of all genetically independent pathways, determination of the most efficient physiological state of a cell, and analysis of metabolic network properties such as robustness and regulation [14-16]. Hence, it can be a useful tool for understanding dynamics of cellular metabolism and rational design of the host strain's metabolism for 2-FL production.

We have previously developed a recombinant $E$. coli system for efficient production of GDP-L-fucose by metabolic engineering. An enhancement of GDPL-fucose production was achieved by modulation of several factors for biosynthesis of GDP-L-fucose such as amplification of GDP-D-mannose biosynthesis, regeneration of NADPH and manipulation of the guanosine nucleotides biosynthetic pathway [17-19].

In the present study, the GDP-L-fucose production system was applied for efficient production of 2-FL by

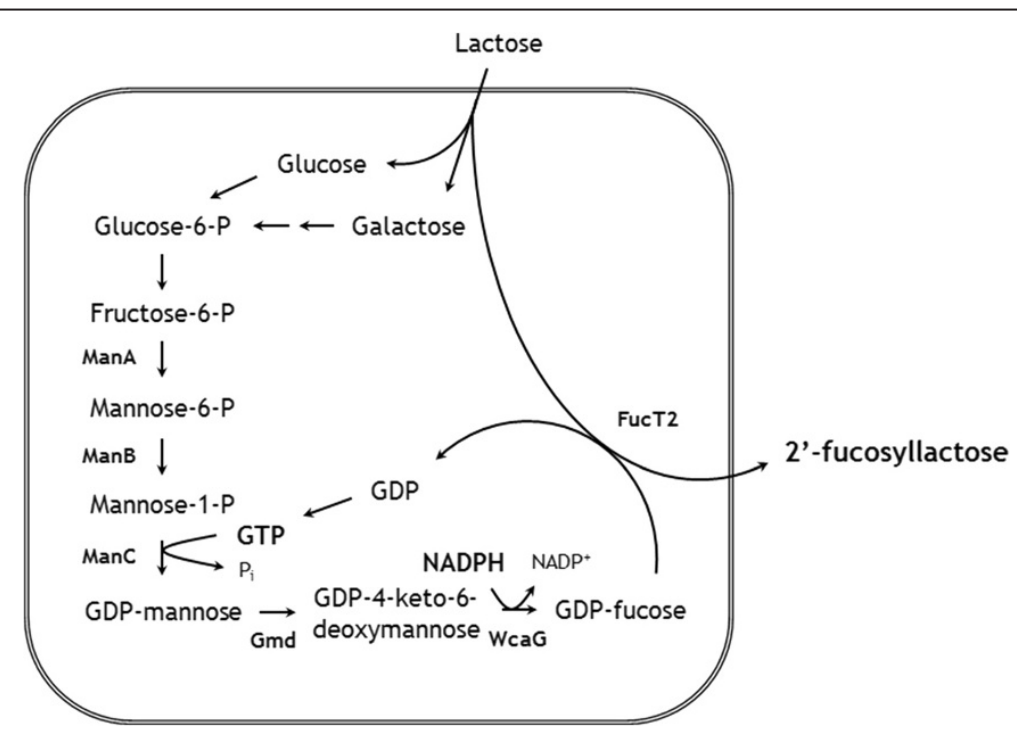

Figure 1 The metabolic pathway for GDP-L-fucose and 2'-fucosyllactose (2-FL) biosynthesis in recombinant $E$. coli. The names of enzymes are abbreviated as follows; ManA, mannose 6-phosphate isomerase; ManB, phosphomannomutase; ManC, mannose 1-phosphate guanylyltransferase; Gmd, GDP-D-mannose-4,6-dehydratase; WcaG, GDP-4-keto-6-deoxymannose 3,5-epimerase 4-reductase; FucT2, a-1,2-fucosyltransferase. Pi, GDP and GTP denote phosphate, guanosine 5'-diphosphate and guanosine 5'-triphosphate. 
introduction of the FucT2 gene from Helicobacter pylori into the recombinant $E$. coli able to overproduce GDP-Lfucose. Whole cell biosynthesis of 2-FL from lactose was assessed in a series of batch fermentations for recombinant E. coli overexpressing the necessary genes for GDPL-fucose production and the FucT2. An EFM analysis for 2-FL production in the recombinant $E$. coli was used to compare and evaluate experimental results.

\section{Methods}

\section{Strains and plasmids}

E. coli TOP10 [F- mcrA $\Delta$ (mrr-hsdRMS-mcrBC)

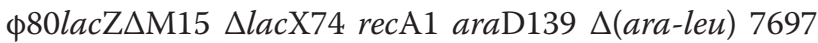
gal $\mathrm{U}$ gal $\mathrm{K}$ rps $\mathrm{L}\left(\mathrm{Str}^{\mathrm{R}}\right)$ end $\mathrm{A} 1$ nup $\left.\mathrm{G}\right]$ was used for genetic manipulation. E. coli $\mathrm{BL} 21 \mathrm{star}(\mathrm{DE} 3)\left[\mathrm{F}^{-}\right.$, omp $\mathrm{T}$, hsd $\mathrm{SB}$ $\left(\mathrm{r}_{\mathrm{B}}^{-} \mathrm{m}_{\mathrm{B}}^{-}\right)$, gal, dcm rne131 (DE3)] (Invitrogen, Carlsbad, CA, USA) and JM109(DE3) [endA1 glnV44 thi-1 relA1 gyr $\mathrm{A} 96$ rec $\mathrm{A} 1$ mcr $^{+} \Delta($ lac-pro $\mathrm{AB})$ e14- $\left[\mathrm{F}^{\prime}\right.$ traD36 pro $\mathrm{AB}^{+}$lacl ${ }^{\mathrm{q}}$ lac $\left.\mathrm{Z} \Delta \mathrm{M} 15\right]$ hsdR17( $\left.\mathrm{r}_{\mathrm{K}}^{-} \mathrm{m}_{\mathrm{K}}^{+}\right)$(DE3)] (NEB, Ipswich, MA, USA) were used for production of GDPL-fucose and 2-FL. Plasmid pmBCGW containing the polycistronic gmd-wcaG gene cluster and manB-manC gene cluster was previously constructed using plasmid pETDuet-1 [18]. The gene encoding FucT2 was obtained by the polymerase chain reactions (PCR) using the genomic DNA of the Helicobacter pylori 26695 strain (ATCC 700392) as template [20]. Two PCR primers of fucT2_F and fucT2_R were used for the amplification of the fucT2 gene. After digestion of PCR fragments of the fucT2 gene and pCOLADuet-1 (Merck Biosciences, Darmstadt, Germany) with NcoI and SacI, the DNA fragments were ligated to construct plasmid pHfucT2. Plasmids and primers used in this work are listed in Table 1. The constructed plasmid was confirmed by DNA sequencing. The conditions for PCR reaction, DNA manipulation and bacterial transformation followed the descriptions in the previous study [21].

\section{Batch fermentation}

Batch fermentation was carried out in a $250 \mathrm{ml}$ flask containing $50 \mathrm{ml}$ of $\mathrm{LB}$ medium at $25^{\circ} \mathrm{C}$ and $\mathrm{pH} 6.8$.
Agitation speed was maintained at $250 \mathrm{rpm}$. When dry cell mass reached $0.3 \mathrm{~g} / \mathrm{l}, \quad 0.1 \mathrm{mM}$ isopropyl- $\beta$-Dthiogalactopyranoside (IPTG) was added to culture broth. After $3 \mathrm{~h}$ of additional cultivation, $2.6 \mathrm{~g} / \mathrm{l}$ (or $14.5 \mathrm{~g} / \mathrm{l})$ lactose was added for 2-FL production.

\section{Analytical methods}

Cell concentration was measured by optical density (OD) at $600 \mathrm{~nm}$ using a spectrophotometer (Biomate 5, Thermo, NY, USA). Overexpression of FucT2 inside the cell was analyzed by using sodium dodecyl sulfate-polyacrylamide gel electrophoresis (SDS-PAGE, $12 \%$ polyacrylamide). After $3 \mathrm{~h}$ of $0.1 \mathrm{mM}$ IPTG induction, cells were collected and the concentration was adjusted to around $7.2 \mathrm{~g} / \mathrm{l}$. They were resuspended in $50 \mathrm{mM}$ potassium phosphate buffer $(\mathrm{pH}$ 7.0) and disrupted by an ultrasonic processor. After centrifugation at $15,000 \times \mathrm{g}$ for $20 \mathrm{~min}$, the supernatant (soluble fraction) and debris (insoluble fraction) were separated. Ten microliters of the soluble protein fraction (approximately $0.04 \mathrm{mg}$ ) and the same volume of the total and insoluble protein fractions were subjected to SDS-PAGE. Gels were stained with Coomassie brilliant blue solution and images were analyzed using a densitometer.

Concentrations of lactose, 2-FL and acetate in batch fermentations were determined by using a high performance liquid chromatography (HPLC) system (Agilent Technologies 1200 Series) equipped with a Rezex ROA Organic Acid $\mathrm{H}^{+}$column (Phenomenex, Torrance, CA, USA) and a refractive index (RI) detector (Agilent, Palo Alto, CA, USA). The column was eluted with $0.01 \mathrm{~N}$ $\mathrm{H}_{2} \mathrm{SO}_{4}$ at a flow rate of $0.6 \mathrm{ml} / \mathrm{min}$ at $50^{\circ} \mathrm{C}$.

In order to confirm 2-FL biosynthesis, culture broth at the end of the batch fermentation was collected and analyzed using a liquid chromatography/mass spectrometry (LC/MS) system. The LC (Agilent Technologies 1100 Series) was equipped with an Agilent Zorbax Eclipse ZDB-C8 (4.6x150 $\mathrm{mm}, 5 \mu \mathrm{m}$ ) column and an Agilent LC/MSD Trap XCT Plus detector. The column was eluted at a flow rate of $0.4 \mathrm{ml} / \mathrm{min}$ by the following gradient program: $95 \%$

Table 1 List of primers and plasmids used in this study

\begin{tabular}{|c|c|c|}
\hline Name & Sequence of PCR primers and description for plasmids & Source \\
\hline \multicolumn{3}{|l|}{ PCR primers } \\
\hline fucT2_F ( NCol) & 5'-ACATGCCATGGCTITTAAGGTGGTGCAA-3' & \multirow{2}{*}{$\begin{array}{l}\text { H. pylori } 26695 \\
\text { (ATCC 700392) }\end{array}$} \\
\hline fucT2_R (SaCl) & 5'-AGTCCGAGCTCTTAAGCGTTATACTTTTGGGA-3' & \\
\hline \multicolumn{3}{|l|}{ Plasmids } \\
\hline pETDuet-1 & two T7 promoters with two MCS, pBR322 replicon (copy number $\sim 40$ ), Ampr & Merck Biosciences \\
\hline pCOLADuet-1 & two T7 promoters with two MCS, ColA replicon (copy number 10 12), Kan ${ }^{r}$ & Merck Biosciences \\
\hline pmBCGW & derived from pETDuet-1, $\mathrm{P}_{\mathrm{T} 7}-$ manB-manC (Ncol/Sacl)- $\mathrm{P}_{\mathrm{T} 7}-$ gmd-wcaG (Ndel/Xhol)- $\mathrm{T}_{\mathrm{T} 7}, \mathrm{Amp}^{\mathrm{r}}$ & Lee et al., 2009 \\
\hline pHfucT2 & derived from pCOLADuet-1, $\mathrm{P}_{\mathrm{T} 7}-$ fucT2 (Ncol/Sacl)-P $\mathrm{P}_{\mathrm{T}}-\mathrm{MCS} 2-\mathrm{T}_{\mathrm{T} 7}, \mathrm{Kan}^{\mathrm{r}}$ & this study \\
\hline
\end{tabular}


(v/v) eluent A (15 mM ammonium acetate) and 5\% eluent B (acetonitrile) for $1 \mathrm{~min}$; $5 \%$ to $95 \%$ eluent B over $6 \mathrm{~min}$; $95 \%$ eluent $\mathrm{B}$ over $10 \mathrm{~min}$. The scan range for MS was 70-600 mass-to-charge ratio $(\mathrm{m} / \mathrm{z})$.

\section{Construction of metabolic network model for $E$. coli producing 2-FL from lactose}

A metabolic network model was constructed for 2-FL producing E. coli that grows on lactose. The E. coli network was based on a model that was introduced by Stelling et al. [22] to examine the relationship between structure and function in metabolic networks. Furthermore, the model has been used for calculating elementary flux modes in previous reports [23,24]. The metabolic network was composed of 108 reactions, which were involved in carbon central metabolism, amino acid synthesis, fatty acid synthesis and biomass production (Additional file 1). The catabolic part of the model included substrate uptake reactions, glycolysis, pentose phosphate pathway, TCA cycle, and excretion of by-products (e.g. acetate, formate, lactate, and ethanol). Previous networks were extended to include the anaplerotic reactions (e.g. malic enzyme and pyruvate oxidase) in addition to parallel pathways for initial acetate metabolism. The anabolic part of the model covers the conversion of precursors into building blocks like macromolecules and biomass. The core E. coli model from Stelling et al. [22] was modified in this research to account for lactose consumption and synthesis of 2-FL. Among the reactions added for 2-FL synthesis, some minor adjustments were made to simplify the model. Lactose was assumed to break down to 2 moles of glucose because galactose can be easily converted into glucose-6-phosphate. ATP was used in place of GTP for energy transfer. As for the mass balance, it should be noted that ADP is formed whenever ATP is consumed for all the metabolic reactions in the network. The mass balance equation on ATP is therefore the negative of the mass balance on ADP and thus the two equations are linearly dependent. Therefore, ADP can be excluded from the model in order to simplify the subsequent EFM calculation. The same is true for other cofactor pairs like NADP/NADPH and NAD/NADH. The EFM pathways in the model were estimated using METATOOL 5.1 $[14,16]$ with Matlab.

\section{Results}

Expression of a-1,2-fucosyltransferase (FucT2) in recombinant $E$. coli

The expression pattern of FucT2 was investigated during a batch fermentation of recombinant $E$. coli harboring plasmid pHfucT2. In order to maximize the expression of the soluble form of FucT2 in the recombinant E. coli, $0.1 \mathrm{mM}$ of IPTG was used. As shown in Figure 2, a
$33 \mathrm{kDa}$ protein (consistent with FucT2, [20]) was found in both soluble and insoluble fraction. While a significant amount of FucT2 was expressed in inclusion bodies, biosynthesis of 2-FL was expected because a soluble form of FucT2 was available as well.

\section{Batch fermentations}

From the preliminary experiments (whole cell bioconversion of $2 \mathrm{~g} / \mathrm{l}$ lactose with E. coli BL21star(DE3) strain), we concluded that the $E$. coli BL21star(DE3) strain is not beneficial for 2-FL production because it consumed lactose for growth and maintenance instead of converting to 2-FL (data not shown). Some E. coli strains, such as $\mathrm{DH} 5 \alpha$ and JM series, are known to be unable to assimilate lactose or utilize lactose extremely inefficiently due to partial deletion of the lac Z gene, which codes for $\beta$-galactosidase. As such, these $E$. coli strains might be useful for 2-FL production. Hence, E. coli JM109(DE3) enabling overexpressing proteins under the control of $T 7$ promoter was chosen as an alternative host stain for 2-FL production.

2-FL production for BL21star(DE3) and JM109(DE3) was compared under batch fermentation conditions. In order to allow sufficient production of both GDPL-fucose biosynthetic enzymes and FucT2 inside the cells, the cells were cultivated for $3 \mathrm{~h}$ after $0.1 \mathrm{mM}$ IPTG induction. Then, $2.6 \mathrm{~g} / \mathrm{l}$ of lactose was added to initiate 2-FL production without addition of additional sugar because GDP-L-fucose can be produced from LB media $[17,18]$. During the fermentations, extracellular 2-FL production (in the medium) was monitored by HPLC analysis. As a result, a small amount of 2-FL (10 mg/l) was produced in the batch fermentation of recombinant $E$. coli BL21star(DE3). Meanwhile, much higher amount of 2-FL was produced in the batch fermentation of recombinant E. coli JM109(DE3). About $140 \mathrm{mg} / \mathrm{l}$ of 2-FL was produced from $2.6 \mathrm{~g} / \mathrm{l}$ of lactose while $0.4 \mathrm{~g} / \mathrm{l}$ of lactose remained unused at the end of the fermentation (data not shown). These results suggest that the lactose concentration should be controlled at more than $0.5 \mathrm{~g} / \mathrm{l}$ to maintain 2-FL production. Consequently, a yield of $60 \mathrm{mg} 2-\mathrm{FL} / \mathrm{g}$ lactose was obtained from the batch fermentation of E. coli JM109(DE3) when $2.6 \mathrm{~g} / \mathrm{l}$ of lactose was used. In order to obtain a higher amount of 2-FL, a batch fermentation with a higher concentration of lactose was carried out. Figure 3 shows the profiles of lactose consumption and 2-FL production in the batch fermentation of recombinant E. coli JM109(DE3) with $14.5 \mathrm{~g} / \mathrm{l}$ lactose. The cells consumed lactose slowly but produced 2-FL constantly for $96 \mathrm{~h}$. After $96 \mathrm{~h}$ of fermentation, the 2-FL concentration did not increase any further and lactose consumption stopped. As a result, a maximum 2-FL concentration of $1.23 \mathrm{~g} / \mathrm{l}$ was obtained, 


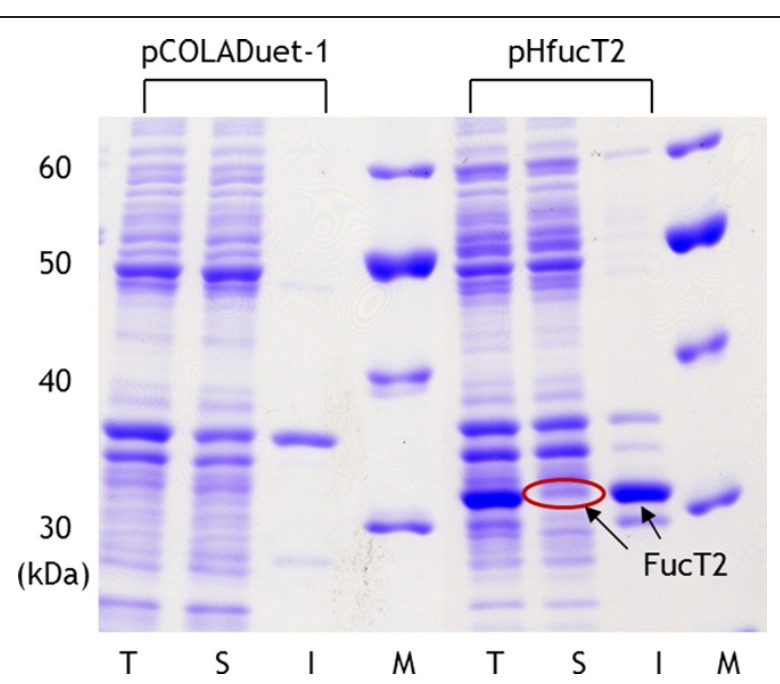

Figure 2 SDS-PAGE analysis of the cell crude extract of recombinant E. coli BL21star(DE3) strains harboring pCOLADuet-1 and pHfucT2, respectively. Cells were harvested after $3 \mathrm{~h}$ of $0.1 \mathrm{mM}$ IPTG induction. T, S and I denote total, soluble and insoluble protein fractions, respectively. The arrow indicates the corresponding protein band with the estimated molecular weight of FucT2. Lane M indicates size marker.

which corresponded to a nine-fold $(1.23 \mathrm{~g} / \mathrm{l}$ vs. $140 \mathrm{mg} / \mathrm{l})$ increase as compared with the previous fermentation with $2.6 \mathrm{~g} / \mathrm{l}$ lactose. 2- FL yield increased to $90 \mathrm{mg} 2-\mathrm{FL} / \mathrm{g}$ lactose when $14.5 \mathrm{~g} / \mathrm{l}$ of lactose was used. This improvement might be caused from increased lactose availability inside the cell. The results of the batch fermentations are summarized in Table 2.
Confirmation of 2-FL biosynthesis by recombinant E. coli overexpressing GDP-L-fucose biosynthetic enzymes and FucT2

LC/MS analysis was performed to confirm the biosynthesis of 2-FL in the recombinant E. coli JM109(DE3) strain overexpressing ManB, ManC, Gmd, WcaG and FucT2. HPLC data showed that a compound with the

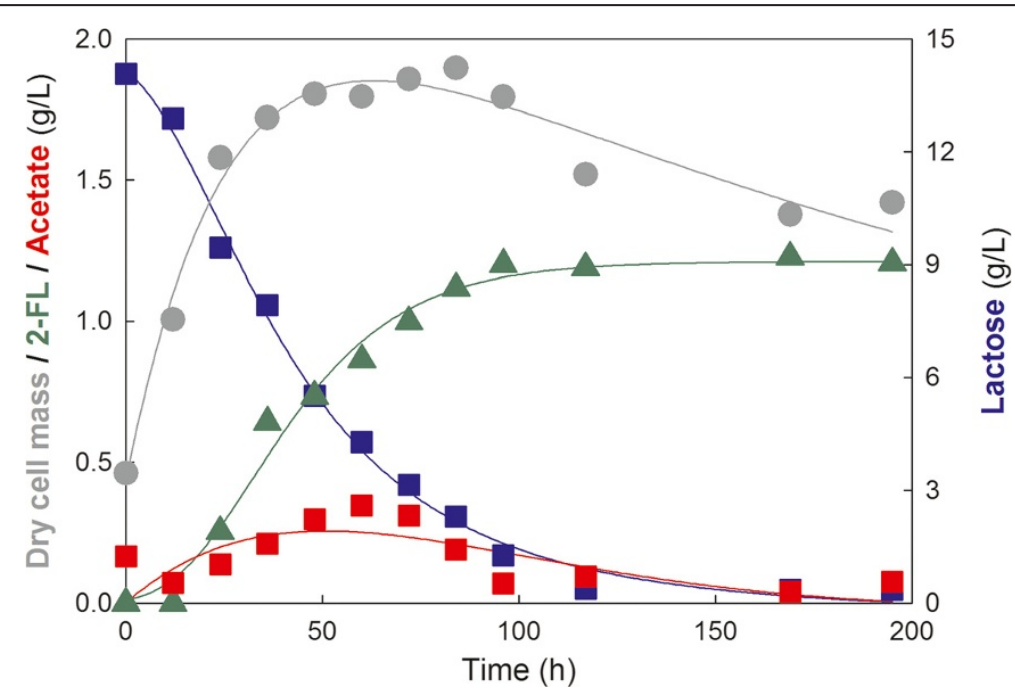

Figure 3 Profile of 2-FL production in the batch fermentation of recombinant E. coli JM109(DE3) strain harboring plasmids pmBCGW and pHfucT2. After $3 \mathrm{~h}$ of $0.1 \mathrm{mM}$ IPTG induction, $14.5 \mathrm{~g} / \mathrm{l}$ of lactose was added for 2-FL production. Symbols denote as follows; grey circle, dry cell mass; green triangle, 2-FL concentration; blue square, lactose concentration; red square, acetate concentration. Measurement of cell, lactose, acetate and 2-FL concentrations were done by three independent experiments. Symbols in the figure show the representative values of the batch fermentations. 
Table 2 Summary of batch fermentations of $E$. coli strains producing 2-FL from lactose

\begin{tabular}{cccccc}
\hline Strains & Plasmids & $\begin{array}{c}\text { Initial lactose } \\
\text { concentration (g/l) }\end{array}$ & $\begin{array}{c}\text { Maximum dry } \\
\text { cell mass (g/l) }\end{array}$ & $\begin{array}{c}\text { Maximum 2-FL } \\
\text { concentration (g/l) }\end{array}$ & $\begin{array}{c}\text { Yield } \\
\text { (g 2-FL/g lactose) }\end{array}$ \\
\hline BL21star(DE3) & pmBCGW + pHfucT2 & $2.56 \pm 0.04$ & $1.84 \pm 0.05$ & $0.01 \pm 0.001$ & $0.005 \pm 0.001$ \\
JM109(DE3) & pmBCGW + pHfucT2 & $2.55 \pm 0.02$ & $1.17 \pm 0.05$ & $0.14 \pm 0.015$ & $0.06 \pm 0.005$ \\
& & $14.54 \pm 0.67$ & $1.70 \pm 0.28$ & $1.23 \pm 0.011$ & $0.09 \pm 0.004$ \\
\hline
\end{tabular}

identical retention time to 2-FL was detected in the culture broth (Figure 4B). MS scanning data (compound with $\mathrm{RT}=6.6 \mathrm{~min}$ ) showed ion fragment of $\mathrm{m} / \mathrm{z} 487.1$, which is compatible with 2-FL (Figure 4C).

\section{Evaluation of 2-FL yield using EFM analysis for 2-FL producing $E$. coli from lactose}

In order to evaluate the efficiency of 2-FL production from lactose using the recombinant E. coli JM109 (DE3) strain, elementary flux mode (EFM) analysis was employed to estimate a maximum theoretical yield of 2-FL from lactose. Figure 5 shows the prediction of theoretical 2-FL yield versus biomass yield for $E$. coli growing on lactose. Our experimental result from a batch fermentation of $14.5 \mathrm{~g} / \mathrm{l}$ of lactose resulted in a biomass yield of $0.1 \mathrm{~g}$ biomass/g lactose. This suggests that 2-FL production from lactose by the engineered E. coli reached $20 \%$ of the maximum 2-FL production capacity.

\section{Discussion}

In human milk, $\alpha$-1,2-fucosylated oligosaccharides such as 2-FL, are known to have protective activity against pathogenic bacteria and their toxins [3,5,9]. Hence, the availability of large amounts of $\alpha-1,2$-fucosylated oligosaccharides would be useful in ready-to-use materials, drugs for fundamental investigations or therapeutic purposes. As biosynthesis of GDP-L-fucose, a key compound for biosynthesis of $\alpha$-1,2-fucosylated oligosaccharides, requires a number of enzymes and cofactors such as NADPH and GTP, a whole-cell conversion approach might be more realistic for industrial production than other chemical or enzymatic approaches [25].

In previous studies, improvements of GDP-L-fucose production in recombinant $E$. coli were attempted by manipulating the pathways enabling GDP-L-fucose biosynthesis from glucose [17-19]. This study was undertaken to upgrade the GDP-L-fucose production system for 2-FL production through additional overexpression of FucT2. To this end, the fucT2 gene from $H$. pylori was cloned and

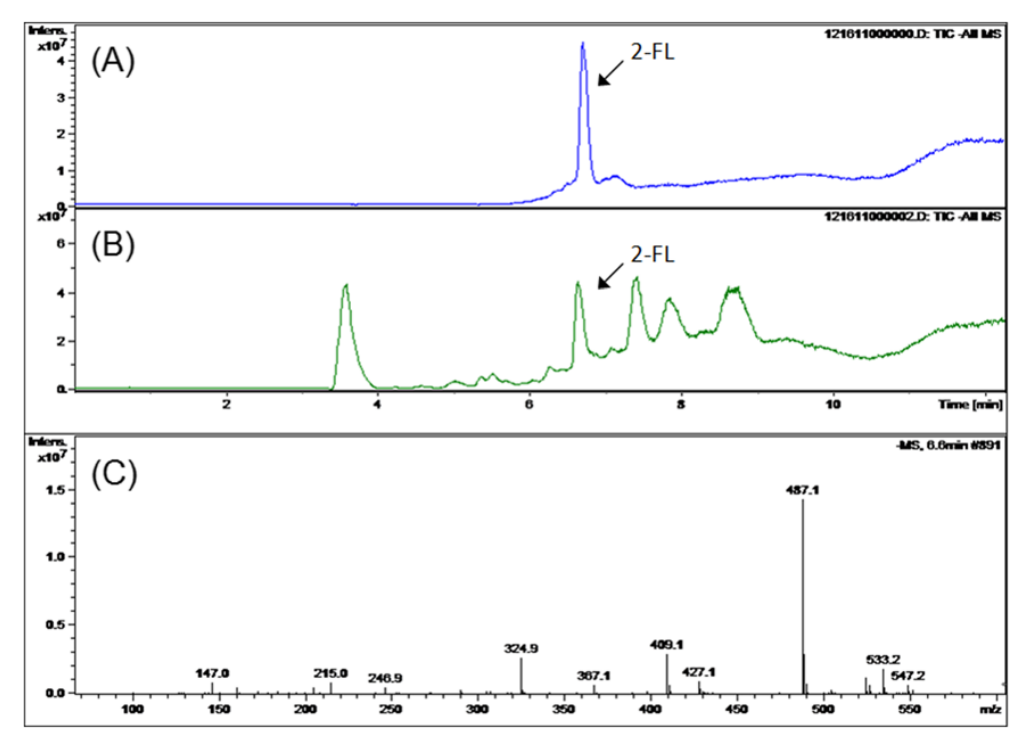

Figure $4 \mathrm{LC} / \mathrm{MS}$ analysis of 2-FL biosynthesis in the batch fermentation of the recombinant E. coli JM109(DE3) overexpressing ManB, ManC, Gmd, WcaG and FucT2. At the end of batch fermentation, culture broth was collected for confirmation of extracellular 2-FL production. HPLC analysis of $100 \mathrm{mg} / \mathrm{l}$ 2-FL standard solution (A), HPLC analysis of culture broth of E. coli JM109(DE3) harboring pmBCGW + pHfucT2 (B) and MS analysis of the compound with the retention time $=6.6 \mathrm{~min}$ in the culture broth of $E$. coli JM109(DE3) harboring pmBCGW + pHfucT2 (C). 


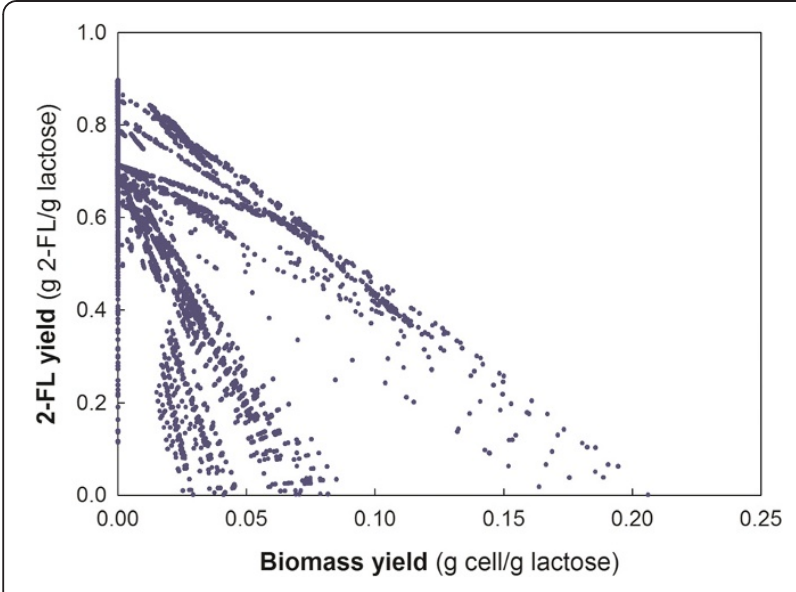

Figure 5 Calculation of the theoretical maximum yield of 2-FL from lactose. Elementary flux mode (EFM) analysis was carried out for 2-FL producing E. coli.

overexpressed in the E. coli BL21star(DE3) strain. While an insoluble form of FucT2 was a major expression form, an active FucT2 was also observed in the recombinant $E$. coli as shown in Figure 2. With the aid of FucT2 overexpression, recombinant E. coli BL21star(DE3) could produce 2-FL in the batch fermentation with $2.6 \mathrm{~g} / \mathrm{l}$ lactose. However, 2-FL yield was fairly low (5 mg 2-FL/g lactose). E. coli BL21star(DE3) seemed to assimilate lactose instead of converting to 2-FL. Most of the initially added lactose was consumed within $12 \mathrm{~h}$ of fermentation with a marginal growth during the fermentation (data not shown). These results suggested that $E$. coli BL21star(DE3) is not appropriate for 2-FL production.

Previously, several attempts for production of fucosylated (or sialylated) oligosaccharides from lactose have been made using the derivative of E. coli JM107 and JM109 since these strains are unable to produce an active $\beta$-galactosidase due to the insertion of the M15 single strand DNA into the lacZ gene [12,13,26,27]. In these cases, glucose (or glycerol) was used as another carbon source for GDP-fucose production (or CMP- $\mathrm{N}$-acetylneuraminic acid). These nucleotide sugars are subsequently used for fucosylation (or sialylation). According to the previous reports, E. coli JM109(DE3) was chosen as an alternative host strain for 2-FL production. As expected, the use of E. coli JM109(DE3) allowed production of a considerable amount of 2-FL in the batch fermentation, corresponding to a 14-fold increase in 2FL concentration compared with the value obtained in BL21star(DE3) strain. However, a theoretical yield of 2-FL from lactose was predicted to be $1.4 \mathrm{~g} 2-\mathrm{FL} / \mathrm{g}$ lactose when cells cannot utilize lactose for growth. Meanwhile, our experimental yield of 2-FL was only about $0.1 \mathrm{~g} 2-\mathrm{FL} / \mathrm{g}$ lactose. This result indicates that more than $90 \%$ of lactose consumed was used for other purposes such as biomass production and endogeneous metabolism. Slow consumption of lactose was also observed in the batch fermentation with mixed sugars ( $2 \mathrm{~g} / \mathrm{l}$ of lactose and $5 \mathrm{~g} / \mathrm{l}$ of mannose), where we expected that lactose could be mainly used for 2-FL production as mannose might be used for cell growth. Although an enhancement of 2-FL yield (0.13 g 2-FL/g lactose) was obtained, most of the consumed lactose was not used for 2-FL production (data not shown). It is probable that incompletely inactivated $\beta$-galactosidase or cryptic $\beta$ galactosidase inside the cell might cause the reduction of 2-FL yield from lactose. Insertion of $\lambda(\mathrm{DE} 3)$ into the genome might cause the slow assimilation of lactose, which was supported by a previous report that $\alpha$-complementation of LacZ was observed with the insertion of the DE3 cassette into the genomic DNA of E. coli JM101 [26].

Since slow consumption of lactose and production of biomass were observed in the batch fermentation with $2.6 \mathrm{~g} / \mathrm{l}$ lactose, another batch fermentation with a high concentration of lactose $(14.5 \mathrm{~g} / \mathrm{l})$ was conducted in order to obtain a higher amount of 2-FL. Generally, $E$. coli is known to produce acetate when growing on an excessive sugar even under aerobic conditions. However, JM109(DE3) strain showed no acetate production even when excessive amounts (14.5 g/l) of lactose were added as displayed in Figure 3. It is generally known that acetate formation is accelerated when the metabolic fluxes to pyruvate exceed the capacity of the respiratory metabolism $[28,29]$. Slow consumption of lactose might not lead to acetate formation, suggesting that the lactose utilization rate by E. coli JM109(DE3) is not fast enough to cause acetate formation. This result could be beneficial for designing a 2-FL production process such as a fed-batch type operation since acetate formation is known to be one of the main problems occurring in fedbatch type operations of E. coli [17]. However, low yield and productivity of 2-FL from lactose will need to be improved for industrial applications. The 2-FL production system in this study was verified by comparing the experimental 2-FL yield with the theoretical maximum yield estimated by the EFM analysis. 2-FL yield corresponded to about $20 \%$ of the theoretical maximum yield, which suggests further modifications via metabolic engineering of a host strain or optimization of fermentation processes should be carried out for improvement of 2-FL yield. Increased FucT2 solubility and intracellular lactose availability may be considered as primary approaches for improvement of 2-FL yield.

\section{Conclusions}

In this study, construction of efficient 2-FL production system was attempted. The fucT2 gene from $H$. pylori was introduced into the recombinant $E$. coli able to overproduce GDP-L-fucose and biosynthesis of 2-FL was 
observed in the batch fermentation with lactose. The 2-FL production system using the E. coli JM109(DE3) strain showed a low rate of lactose assimilation and produced a considerable amount of 2-FL in the simple batch fermentation without acetate formation. The experimental 2-FL yield corresponded to $20 \%$ of the theoretical maximum yield, which indicates that more research should be conducted. Efficient microbial 2-FL production may be useful for utilizing 2-FL as a nutraceutical compound for various applications.

\section{Additional file}

\section{Additional file 1: METATOOL input file.}

\section{Competing interests}

The authors declare that they have no competing interests.

\section{Authors' contributions}

WHL, YSJ and JHS designed research. WHL, PP, JQ and JHJ performed the experiments. NSH and MJM contributed general advice and materials. WHL, PP, JQ, YSJ and JHS analyzed data and wrote the manuscript. YSJ and JHS supervised all works. All authors read and approved the final manuscript.

\section{Acknowledgements}

This research was supported by World Class University (WCU) program (R32-2008-000-10183-0) and the Advanced Biomass R\&D Center (ABC) (2011-0031359) both funded by the Korean Ministry of Education, Science and Technology. Won-Heong Lee was supported by National Research Foundation of Korea Grant funded by the Korean Government (Ministry of Education, Science and Technology) (NRF-2011-357-F00041). Panchalee Pathanibul was funded by the Royal Thai government scholarship. LC/MS analysis was assisted by Metabolomics Center, Roy J. Carver Biotechnology Center of University of Illinois at UrbanaChampaign.

\section{Author details \\ 'Department of Food Science and Human Nutrition, University of Illinois at Urbana-Champaign, Urbana IL 61801, USA. ${ }^{2}$ Institute for Genomic Biology, University of Illinois at Urbana-Champaign, Urbana IL 61801, USA. ${ }^{3}$ Department of Food Science and Technology, Chungbuk National University, Cheongju 361-763, Korea. ${ }^{4}$ Department of Agricultural Biotechnology and Center for Agricultural Biomaterials, Seoul National University, Seoul 151-921, Korea.}

Received: 15 February 2012 Accepted: 17 April 2012 Published: 30 April 2012

\section{References}

1. Kunz C, Rudloff S: Health promoting aspects of milk oligosaccharides. Int Dairy J 2006, 16(11):1341-1346.

2. Bode $L$ : Recent advances on structure, metabolism, and function of human milk oligosaccharides. J Nutr 2006, 136(8):2127-2130.

3. Morrow AL, Ruiz-Palacios GM, Altaye M, Jiang X, Guerrero ML, Meinzen-Derr JK, Farkas T, Chaturvedi P, Pickering LK, Newburg DS: Human milk oligosaccharides are associated with protection against diarrhea in breast-fed infants. J Pediatr 2004, 145(3):297-303.

4. Newburg DS, Ruiz-Palacios GM, Altaye M, Chaturvedi P, Meinzen-Derr J, Guerrero MD, Morrow AL: Innate protection conferred by fucosylated oligosaccharides of human milk against diarrhea in breastfed infants. Glycobiology 2004, 14(3):253-263.

5. Chaturvedi P, Warren CD, Altaye M, Morrow AL, Ruiz-Palacios G, Pickering LK Newburg DS: Fucosylated human milk oligosaccharides vary between individuals and over the course of lactation. Glycobiology 2001, 11(5):365-372.
6. Chessa D, Winter MG, Jakomin M, Bäumler AJ: Salmonella enterica serotype Typhimurium Std fimbriae bind terminal $a(1,2)$ fucose residues in the cecal mucosa. Mol Microbiol 2009, 71(4):864-875.

7. Newburg DS, Pickering LK, McCluer RH, Cleary TG: Fucosylated oligosaccharides of human milk protect suckling mice from heat-stabile enterotoxin of Escherichia coli. J Infect Dis 1990, 162(5):1075-1080.

8. Maqalhães A, Reis CA: Helicobacter pylori adhesion to gastric epithelial cells is mediated by glycan receptors. Braz J Med Biol Res 2010, 43 (7):611-618.

9. Newburg DS, Ruiz-Palacios GM, Morrow AL: Human milk glycans protect infants against enteric pathogens. Annu Rev Nutr 2005, 25:37-58.

10. Albermann C, Piepersberg W, Wehmeier UF: Synthesis of the milk oligosaccharide 2'-fucosyllactose using recombinant bacterial enzymes. Carbohydr Res 2001, 334(2):97-103.

11. Stevenson G, Andrianopoulos K, Hobbs M, Reeves PR: Organization of the Escherichia coli K-12 gene cluster responsible for production of the extracellular polysaccharide colanic acid. J Bacteriol 1996, 178 (16):4885-4893.

12. Drouillard $\mathrm{S}$, Driguez $\mathrm{H}$, Samain E: Large scale synthesis of $H$ antigen oligosaccharides by expressing Helicobacter pylori a1, 2-fucosyltransferase in metabolically engineered Escherichia coli cells. Angewandte Chemie 2006, 118(11):1810-1812

13. Dumon C, Priem B, Martin SL, Heyraud A, Bosso C, Samain E: In vivo fucosylation of lacto- $N$-neotetraose and lacto- $N$-neohexaose by heterologous expression of Helicobacter pylori a-1, 3 fucosyltransferase in engineered Escherichia coli. Glycoconj J 2001, 18(6):465-474.

14. Pfeiffer T, Sánchez-Valdenebro I, Nuño JC, Montero F, Schuster S: METATOOL: for studying metabolic networks. Bioinfomatics 1999, 15(3):251-257.

15. Schuster S, Fell DA, Dandekar T: A general definition of metabolic pathways useful for systematic organization and analysis of complex metabolic networks. Nat Biotechnol 2000, 18(3):326-332.

16. Trinh $C T$, Wlaschin A, Srienc F: Elementary mode analysis: a useful metabolic pathway analysis tool for characterizing cellular metabolism. Appl Microbiol Biotechnol 2009, 81(5):813-826.

17. Lee WH, Chin YW, Han NS, Kim MD, Seo JH: Enhanced production of GDP-L-fucose by overexpression of NADPH regenerator in recombinant Escherichia coli. Appl Microbiol Biotechnol 2011, 91(4):967-976.

18. Lee WH, Han NS, Park YC, Seo JH: Modulation of guanosine $5^{\prime}$-diphosphate-D-mannose metabolism in recombinant Escherichia coli for production of guanosine $5^{\prime}$-diphosphate-L-fucose. Bioresour Technol 2009, 100(24):6143-6148.

19. Lee WH, Shin SY, Kim MD, Han NS, Seo JH: Modulation of guanosine nucleotides biosynthetic pathways enhanced GDP-L-fucose production in recombinant Escherichia coli. Appl Microbiol Biotechnol 2012, 93(6):2327-2334

20. Wang G, Boulton PG, Chan NW, Palcic MM, Taylor DE: Novel Helicobacter pylori a1,2-fucosyltransferase, a key enzyme in the synthesis of Lewis antigens. Microbiology 1999, 145(11):3245-3253.

21. Byun SG, Kim MD, Lee WH, Lee KJ, Han NS, Seo JH: Production of GDP-L-fucose, L-fucose donor for fucosyloligosaccharide synthesis, in recombinant Escherichia coli. Appl Microbiol Biotechnol 2007, 74(4):768-775.

22. Stelling J, Klamt S, Bettenbrock K, Schuster S, Gilles ED: Metabolic network structure determines key aspects of functionality and regulation. Nature 2002, 420(6912):190-193.

23. Klamt S, Gagneur J, von Kamp A: Algorithmic approaches for computing elementary modes in large biochemical reaction networks. IEE Proc Syst Biol 2005, 152(4):249-255.

24. Urbanczik R, Wagner C: An improved algorithm for stoichiometric network analysis: theory and applications. Bioinformatics 2005, 21(7):1203-1210.

25. Ruffing A, Chen RR: Metabolic engineering of microbes for oligosaccharide and polysaccharide synthesis. Microb Cell Fact 2006, 5:25

26. Dumon C, Samain E, Priem B: Assessment of the two Helicobacter pylori a-1, 3 fucosyltransferase ortholog genes for the large scale synthesis of LewisX human milk oligosaccharides by metabolically engineered Escherichia coli. Biotechnol Prog 2004, 20(2):412-419.

27. Fierfort N, Samain E: Genetic engineering of Escherichia coli for the economical production of sialylated oligosaccharides. J Biotechnol 2008, 134(3-4):261-265. 
28. Lee SY: High cell-density culture of Escherichia coli. Trends Biotechnol 1996, 14(3):98-105.

29. Zhao J, Baba T, Mori H, Shimizu K: Global metabolic response of Escherichia coli to gnd or zwf gene-knockout, based on ${ }^{13} \mathrm{C}$-labeling experiments and the measurement of enzyme activities. App/ Microbiol Biotechnol 2004, 64(1):91-98.

doi:10.1186/1475-2859-11-48

Cite this article as: Lee et al: Whole cell biosynthesis of a functional oligosaccharide, 2'-fucosyllactose, using engineered Escherichia coli. Microbial Cell Factories 2012 11:48.

\section{Submit your next manuscript to BioMed Central} and take full advantage of:

- Convenient online submission

- Thorough peer review

- No space constraints or color figure charges

- Immediate publication on acceptance

- Inclusion in PubMed, CAS, Scopus and Google Scholar

- Research which is freely available for redistribution 